\title{
Micro-transfer-printed III-V-on-silicon C-band Distributed Bragg Reflector Laser
}

\author{
Jing Zhang \\ Ghent University - imec \\ Ghent, Belgium \\ jingzhan.zhang@ugent.be \\ Agnieszka Gocalinska \\ Tyndall National Institute \\ Cork, Ireland \\ agnieszka.gocalinska@tyndall.ie
}

\author{
Sulakshna Kumari \\ Ghent University - imec \\ Ghent, Belgium \\ sulakshna.kumari@ugent.be
}

\author{
Emanuele Pelucchi \\ Tyndall National Institute \\ Cork, Ireland
}

emanuele.pelucchi@tyndall.ie

\author{
Sören Dhoore \\ Ghent University - imec \\ Ghent, Belgium \\ soren.dhoore@ugent.be \\ Brian Corbett \\ Tyndall National Institute \\ Cork, Ireland \\ brian.corbett@tyndall.ie
}

\author{
Grigorij Muliuk \\ Ghent University - imec \\ Ghent, Belgium \\ grigorij.muliuk@ugent.be \\ Gunther Roelkens \\ Ghent University - imec \\ Ghent, Belgium \\ gunther.roelkens@ugent.be
}

\begin{abstract}
We demonstrate a micro-transfer-printed III-Von-silicon distributed Bragg reflector (DBR) laser using preprocessed III-V semiconductor optical amplifiers (SOAs). A waveguide-coupled output power of $6 \mathrm{~mW}$ is obtained at 1565 nm.

Index Terms-micro-transfer-printing, III-V-on-silicon heterogeneous integration, lasers
\end{abstract}

\section{INTRODUCTION}

The integration of III-V semiconductors on silicon photonic integrated circuits is necessary to implement gain and therefore lasers on the silicon photonics platform. This III-V integration can be done in several ways, including flip-chip or pickand-place integration as well as die-to-wafer or wafer-towafer bonding. Flip-chip/pick-and-place processes however are sequential in nature and therefore do not allow for a high throughput integration [1]. On the other hand these techniques allow the integration of pre-fabricated and pre-tested devices. Die-to-wafer bonding or wafer-to-wafer bonding allows for high throughput integration as the III-V processing is done on wafer level [2]. However, besides requiring the capability to process III-V devices on $200 \mathrm{~mm} / 300 \mathrm{~mm}$ wafers, there is a potential compound yield issue as the lasers can only be tested after they have been integrated on the silicon photonic wafer. Moreover, the silicon photonics back-end process flow needs to be adapted to accommodate the III-V devices. Recently, we have started the development of a novel technique, microtransfer-printing [3], to integrate III-V devices on a silicon photonic wafer. The process is schematically illustrated in Fig. 1. In this technique, the III-V opto-electronic components are pre-fabricated on the III-V source wafer. This is done by incorporating a release layer in the epitaxial layer stack (InAlAs), such that after device processing the devices can be encapsulated and released from the InP substrate by wet etching of the release layer. This leaves the devices free-standing on the InP substrate, solely held in place by the patterned

This work was supported by the H2020 TOPHIT project and by the Methusalem funding of the Flemish government. tethers. These devices can be picked-up by a PDMS stamp and printed on the silicon photonic target wafer, after which the encapsulation is removed and the device is electrically connected to the back-end stack. This technique combines advantages of flip-chip/pick-and-place integration (namely the integration of pre-fabricated, pre-tested devices) and wafer bonding approaches (the high throughput integration, as large arrays of III-V devices can simultaneously be integrated). In this paper we elaborate on the demonstration of the technique for realizing III-V-on-silicon distributed Bragg reflector (DBR) lasers in the C-band.

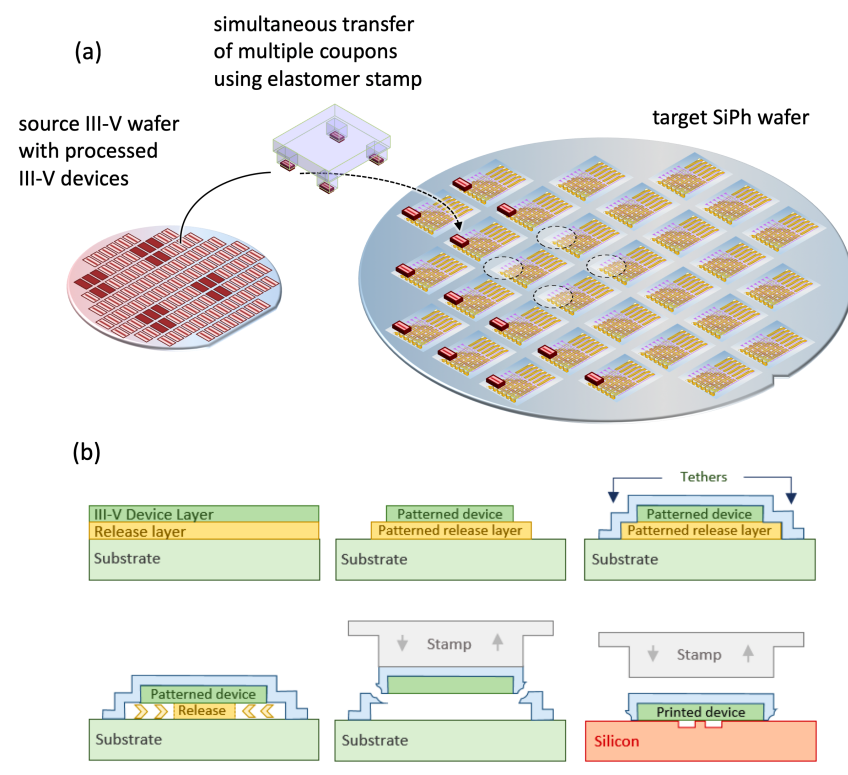

Fig. 1. Schematic of the micro-transfer-printing III-V-on-silicon heterogeneous integration process: (a) overview of wafer-level integration process; (b) zoom-in on the III-V fabrication process and printing operation.

\section{DEVICE FABRICATION AND CHARACTERIZATION}

The III-V processing comprises the standard processing steps for the realization of III-V semiconductor optical ampli- 
fiers (SOAs) used in heterogeneous III-V-on-silicon devices, including III-V mesa etching using a combination of wet and dry chemical etching, the side n-metal definition, the planarization using DVS-BCB and the p-metal definition and via opening to the n-metal. While in die-to-wafer or waferto-wafer bonding approaches this is realized on the bonded epitaxial film, in micro-transfer-printing the fabrication is done - in dense arrays - on the III-V source wafer, that incorporates a $500 \mathrm{~nm}$ InAlAs release layer between the InP substrate and the III-V laser epitaxy (consisting of a $260 \mathrm{~nm}$ thick n-InP, an InAlGaAs active region with 6 quantum wells, and a 2 $\mu \mathrm{m} / 285 \mathrm{~nm}$ thick $\mathrm{p}-\mathrm{InP} / \mathrm{p}$-InGaAs cladding). Taper structures are defined in the III-V waveguide layer to allow for the efficient optical coupling with the silicon waveguide layer. After amplifier fabrication, the $500 \mathrm{~nm}$ InAlAs release layer is patterned and devices are encapsulated with photoresist, which will protect the devices during the release etch and keeps the devices in place after the release layer has been etched. A $\mathrm{FeCl}_{3}: \mathrm{H}_{2} \mathrm{O}$ etch is used to selectively etch the InAlAs release layer. A microscope image of released III-V semiconductor optical amplifiers is shown in Fig. 2.

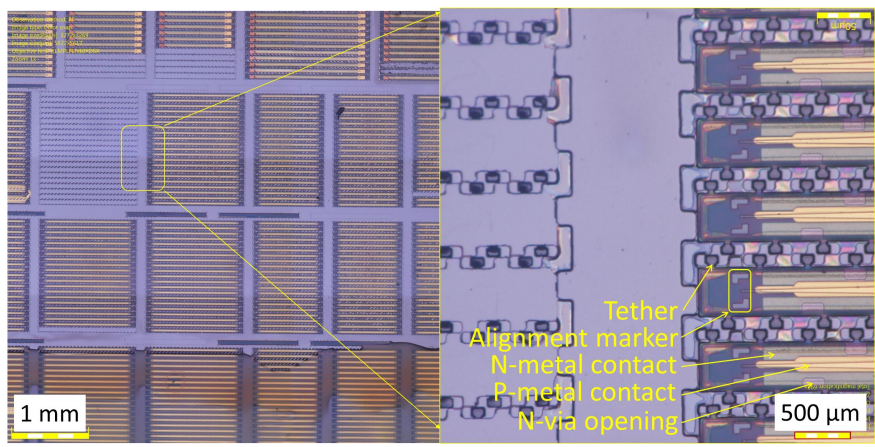

Fig. 2. Microscope image of released III-V semiconductor optical amplifiers on the source substrate. One column of devices has already been microtransfer-printing. Only the broken tether remain there.

The SOAs, with a length of $960 \mu \mathrm{m}$, excluding the 2 tapers of $200 \mu \mathrm{m}$, and a mesa width of $4.5 \mu \mathrm{m}$, are microtransfer-printed using a $20 \mathrm{~nm}$ thick DVS-BCB bonding layer on a silicon-on-insulator wafer with $400 \mathrm{~nm}$ silicon device layer thickness and $2 \mu \mathrm{m}$ buried oxide, in which $180 \mathrm{~nm}$ etched waveguide structures are defined. The laser cavity is $1800 \mu \mathrm{m}$ long and consists of a rear DBR and front DBR realized by corrugating the width of the silicon waveguide (period $247 \mathrm{~nm}, 900$ periods and 600 periods for the rear and front DBR respectively). After the micro-transfer-printing the photoresist encapsulation is removed and the device is electrically contacted. A microscope image of micro-transferprinted and post-processed SOAs on the Si photonic circuit is shown in the inset of Fig. 4.

The III-V-on-silicon DBR lasers are characterized on a temperature-controlled stage at $20^{\circ} \mathrm{C}$. The L-I-V curve (waveguide-coupled output power) is shown in Fig. 3. The series resistance of the device is $6 \Omega$. A laser threshold of 140
$\mathrm{mA}$ and a maximum waveguide-coupled output power over 6 $\mathrm{mW}$ is obtained. Fig. 4 shows a recorded spectrum at $290 \mathrm{~mA}$, showing laser emission around $1565 \mathrm{~nm}$.

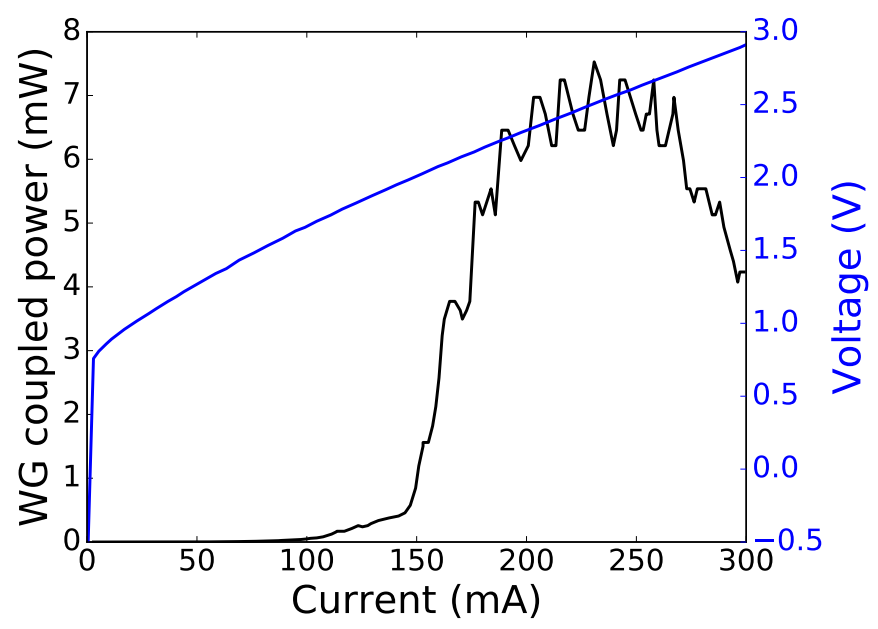

Fig. 3. P-I-V curve of the micro-transfer-printed III-V-on-silicon DBR laser.

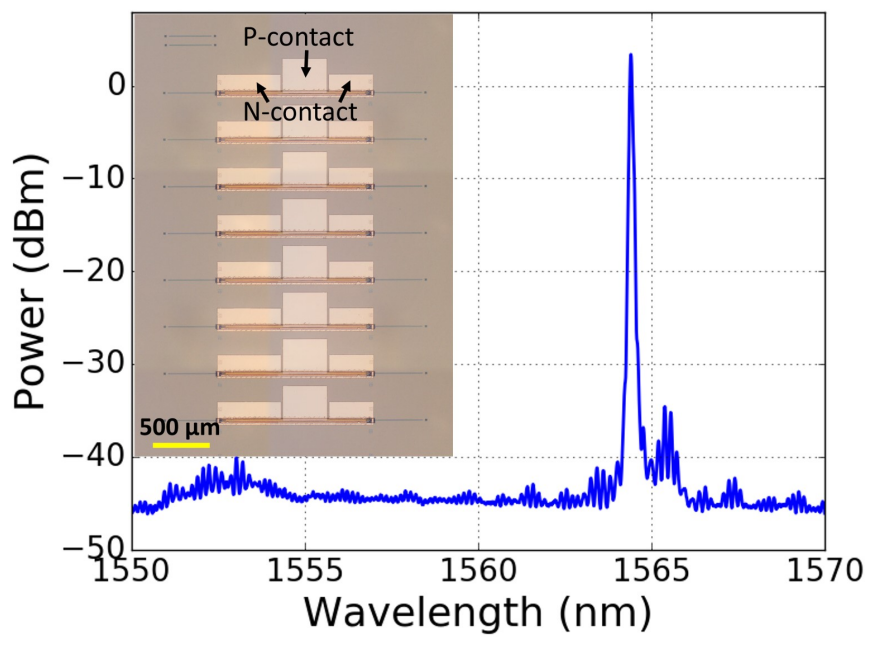

Fig. 4. DBR laser spectrum for a bias current of $290 \mathrm{~mA}$. Inset: a microscope image of an array of fabricated DBR lasers.

\section{CONCLUSION}

In this paper we demonstrated a III-V-on-silicon DBR laser using micro-transfer-printing of pre-fabricated III-V semiconductor optical amplifiers on a silicon waveguide circuit. This demonstration showcases the great potential that the microtansfer-printing technique has for the realization of III-V-onsilicon photonic integrated circuits.

\section{REFERENCES}

[1] S. Tanaka, et al.,Fujitusu. Sci. Tech. J., 50(1), pp.123131, 2014.

[2] X. Luo, et al.,Front. Mater., vol.2, pp.28, 2015.

[3] E. Menard, et al.,Appl. Phys. Lett., 84(26), pp.5398-5400, 2004. 